\title{
Synthesis and characterization of oleic acid stabilized CdTe quantum dots and their properties as luminescence quencher of a pyridine pendented rod-coil homopolymer
}

\author{
MANPREET KAUR ${ }^{1,2}$, DURYOdHAN SAHU ${ }^{2, *}$ \\ ${ }^{1}$ Department of Nanotechnology Sri Guru Granth Sahib World University Fatehgarh Sahib-140406, Punjab, India \\ ${ }^{2}$ Department of Chemistry, National Institute of Science and Technology, Berhampur, 761008, Odisha, India
}

\begin{abstract}
We report a facile one-step non aqueous synthesis of oleic acid stabilized cadmium telluride (CdTe) quantum dots (QDs) with an average diameter of $3 \mathrm{~nm}$ to $4 \mathrm{~nm}$ by hot injection method. The synthesized oleic acid capped QDs observed by TEM were nearly spherical. The optical properties of QDs were characterized by UV-Vis absorption spectra and photoluminescence (PL) spectra. The structures of QDs and their surface passivation were further verified using transmission electron microscope (TEM), X-ray diffraction (XRD) and Fourier transform infrared spectroscopy (FT-IR). The quenching effect of the CdTe QD was explored by addition of CdTe nanocrystals into a solution of rod-coil homopolymer (poly[10-(6-(9,9-diethyl-7-(pyridin-4yl)-9H-fluoren-2-yl)naphthalen-2-yloxy) decyl methacrylate]) (PFNA) having pendent pyridine. The gradual addition of quantum dots to the solution of PFNA quenched the PL spectra of PFNA. This may be used to explore the coordination ability of pyridine containing homopolymer with CdTe quantum dots.
\end{abstract}

Keywords: CdTe quantum dot; surface passivation; PL quenching; nanocomposite; polymer

\section{Introduction}

Semiconductor quantum dots of different shapes with the radius below their Bohr exciton radius have attracted immense attention due to their outstanding photophysical properties and potential applications in various fields such as lasers, photoluminescence quenching, drug delivery, LED displays photovoltaics etc. [1-4]. Since the development of different synthetic procedures by bottom up approach, quantum dots have become the dependable alternative to the organic fluorophores [5]. Furthermore, luminescence properties of II-VI nanocrystals with tunable sizes between $2 \mathrm{~nm}$ to $7 \mathrm{~nm}$ have exceptional photochemical and photophysical properties [6]. It is wellknown that a chromophoric material near a metal film shows varieties of luminescence depending on the distance from the metal surface and the binding mechanism. Many metals similar to gold which can bring about efficient photoluminescence

*E-mail: duryosahu@gmail.com quenching [7, 8] can be applied in biosensors and chemosensors.

For the last two decades, various quantum dots (II-VI, III-V or IV-VI groups of periodic table) have been synthesized. However, cadmium telluride (CdTe) has drawn considerable attention owing to its wide application potential in electrooptical applications such as photovoltaics, sensors, biomedical applications etc. $[9,10]$. CdTe has potential properties, which include ideal energy band gap of $1.45 \mathrm{eV}$ to $1.5 \mathrm{eV}$ and absorption coefficient of $5.1 \times 10^{5} \mathrm{~cm}^{-1}$ [11]. To make good quality optoelectronic devices it is important to minimize light scattering in composite materials. The low band of CdTe allows us to consider it as an emerging material to be used in electro-optical applications. In addition, the use of appropriate surfactant in the preparation of nanostructures can reduce the number of dangling bonds on the nanoparticle surface, which are believed to weaken the surfacerelated non-radiative recombination and enhance the band-edge luminescence efficiency. Organic capping of the semiconductor QDs provides the 
control on their composition, size, structure and shape. The obvious advantage of CdTe, such as much better electron mobility, results in the replacement of other nanomaterials in optoelectronic devices [12, 13]. The high thermal, photochemical stability and surface passivation through different functional groups make this quantum dot an appealing material for the construction of various optoelectronic devices including photoactive and sensitive molecule-nanocrystals hybrids [14-16].

In this report we synthesized oleic acid stabilized CdTe quantum dots with the size of $3 \mathrm{~nm}$ to $4 \mathrm{~nm}$ through hot injection method and they were characterized by UV-Vis absorption spectra and photoluminescence (PL) spectra. The structure size and the surface passivation of QDs were verified through transmission electron microscope (TEM), X-ray diffraction (XRD) and Fourier transform infrared spectroscopy (FT-IR), respectively. Furthermore, we synthesized a well-known homopolymer (PFNA) containing fluorene core followed by naphthalene and pendent pyridine and sensitized the polymer by addition of oleic acid stabilized CdTe quantum dot solution. The gradual addition of quantum dots caused photoluminescence quenching of the homopolymer (PFNA) owing to the coordination ability of pyridine containing homopolymer with CdTe quantum dots.

\section{Experimental}

\subsection{Materials}

The chemicals used to synthesize the polymer (PFNA) were purchased from Aldrich, ACROS, TCI, Strem and Fluka. The materials used for the preparation of CdTe nanocrystals, such as tellurium powder (200 mesh, $99.8 \%$ ), 1-octadecane (ODE) (90\%), oleic acid, octadecylamine (ODA) (97\%), trioctylphosphine (TOP) (90\%), cadmium acetate dihydrate $(98 \%)$ were purchased from Aldrich. Tetrahydrofuran (THF) and dichloromethane (DCM) were distilled over sodium/benzophenone and calcium hydride respectively; ethanol, N-methyl-2-pyrrolidone, and toluene were reagent grade and used without any further purification.

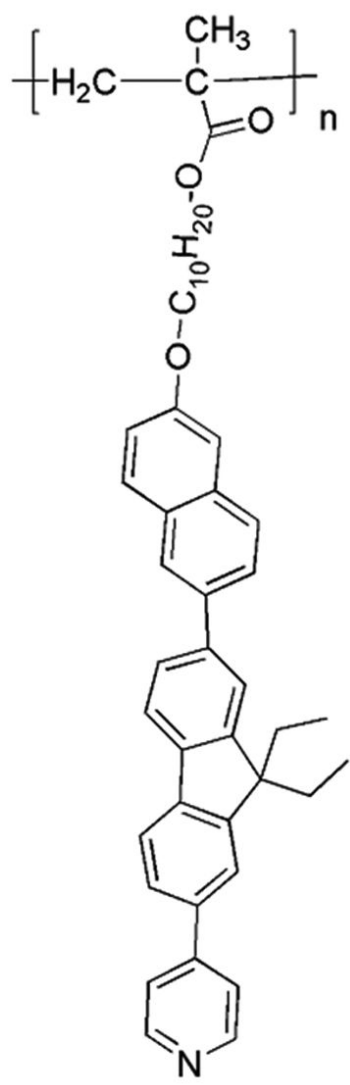

Fig. 1. Structure of rod coil homopolymer (PFNA).

\subsection{Materials synthesis.}

\section{Preparation of CdTe quantum dots}

Colloidal CdTe quantum dots were synthesized via an organic-inorganic route using oleic acid as a stabilizer and ODE as a solvent. $0.55 \mathrm{~g}$ of ODA was placed in $10 \mathrm{ml}(\mathrm{ODE})$ of $0.1 \mathrm{M}$ cadmium acetate solution in a round bottom flask. The above solutions were heated at $110{ }^{\circ} \mathrm{C}$ for $10 \mathrm{~min}$ and then the temperature was raised to $240{ }^{\circ} \mathrm{C}$ followed by addition of $0.283 \mathrm{~g}$ of oleic acid capping agent. To this mixture, $10 \mathrm{ml}$ of $0.1 \mathrm{M}$ Te solution in trioctylphosphine (TOP) was added slowly and the temperature was lowered to $200{ }^{\circ} \mathrm{C}$. The above mixture was stirred for 15 min under $\mathrm{pH} 8$ (could be maintained by addition of $0.5 \mathrm{~mol} / \mathrm{l}$ of $\mathrm{NaOH})$. In this case, ODA acted as reducing agent which reduced the compound to cadmium acetate and oleic acid caped it. The acid capped cadmium nanoparticles formed bonds with Te after addition of Te stock solution into the reaction flask. 


\section{Synthesis of PFNA}

The rod-coil homopolymer PFNA (Fig. 1) was synthesized by the known literature procedure [17]. The chemical structures of the synthesized products were confirmed by ${ }^{1} \mathrm{H}$ NMR spectroscopy, mass spectra (FAB) and elemental analysis. The molecular weight of the polymer was calculated through gel permeation chromatography (GPC). The molecular weight of homopolymer (PFNA) was found to be $15130 \mathrm{~g} / \mathrm{mol}$ with polydispersity index 1.13. The extended decoxy groups in the conjugated segments imparted the resulting polymers with excellent solubility in common organic solvents, such as hexane, dichloromethane, toluene, ethyl acetate, THF, etc. The polymers showed good thermal stability with decomposition temperature of $359{ }^{\circ} \mathrm{C}$ and exhibited stable luminescent characteristics.

\subsection{Characterizations}

Oleic acid capped CdTe nanomaterials were characterized by X-ray diffractometer (powder method), PANalytical X'Pert Pro $(\mathrm{CuK} \alpha$ radiation, variable entrance slit, Bragg-Brentano geometry, secondary monochromator). Samples for these measurements were prepared by placing finely dispersed powders of CdTe nanoparticles on standard PVC supports. TEM studies were performed on a transmission electron microscope (TEM) Hitachi (H-7500) operating at $100 \mathrm{kV}$. TEM samples were prepared by dropping diluted solutions of CdTe nanoparticles onto 400-mesh carbon-coated copper grids where the excess solvent evaporated immediately. The absorbance spectrum was obtained by Schimadzu UV spectrophotometer in the wavelength range of $800 \mathrm{~nm}$ to $200 \mathrm{~nm}$ in absorbance mode using a quartz cuvette. Photoluminescence studies were conducted on Edinburg photoluminescence spectrophotometer FLS 980, FT-IR characterization has been done on Bruker FT-IR.

\section{Results and discussion}

The synthesized non aqueous oleic acid stabilized CdTe quantum dots were obtained as redissolvable cluster powders through centrifugation and subsequent drying in an oven. X-ray

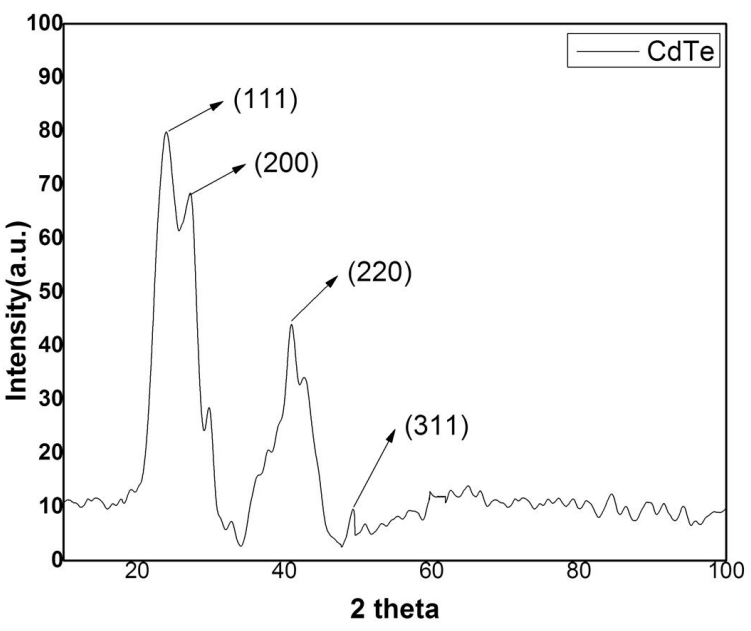

Fig. 2. XRD spectra of oleic acid caped CdTe quantum dots.

diffraction analyses were employed to evaluate the crystalline and structural properties of the powder sample. As shown in Fig. 2, the XRD pattern of the sample has broad peaks at $2 \theta$ values of $24^{\circ}$, $40^{\circ}$, and $48^{\circ}$. All the peak positions in the XRD pattern are clearly indexed with bulk CdTe cubic structure and are consistent with JCPDS Card No. 65-1046. The diffraction angles $(2 \theta), 24^{\circ}, 27^{\circ}, 40^{\circ}$, and $48^{\circ}$ can be assigned to (1 111$),\left(\begin{array}{lll}2 & 0 & 0\end{array}\right),\left(\begin{array}{lll}2 & 2 & 0\end{array}\right)$ and (3 111 ) planes, respectively, corresponding to the cubic zinc blend structure [18]. The diffraction pattern displays typical low intensity/broad peaks of a nanosized material which could be attributed to the finite crystallite size of the nanomaterials [19].

As shown in Fig. 3, the TEM studies reveal that the CdTe nanocrystals exhibit polycrystalline cubic phase with an average size of grains of $3 \mathrm{~nm}$ to $4 \mathrm{~nm}$ and the Bohr exciton radius is much smaller than the bulk CdTe. A strong size quantization effect may manifest itself in so small nanocrystals where only few electronic energy levels exist within the band gap which results in discreteness of energy states with shifting of electronic excitation states to higher energy. As shown in the TEM image of oleic acid stabilized CdTe QDs (Fig. 3), the average size of the QDs is $3.5 \mathrm{~nm}$. As shown in HR TEM micrograph (Fig. 3b inset), the interplanar spacing (encircled in the inset image) was found to be $0.3 \mathrm{~nm}$ which corresponds to (llll) reflections 


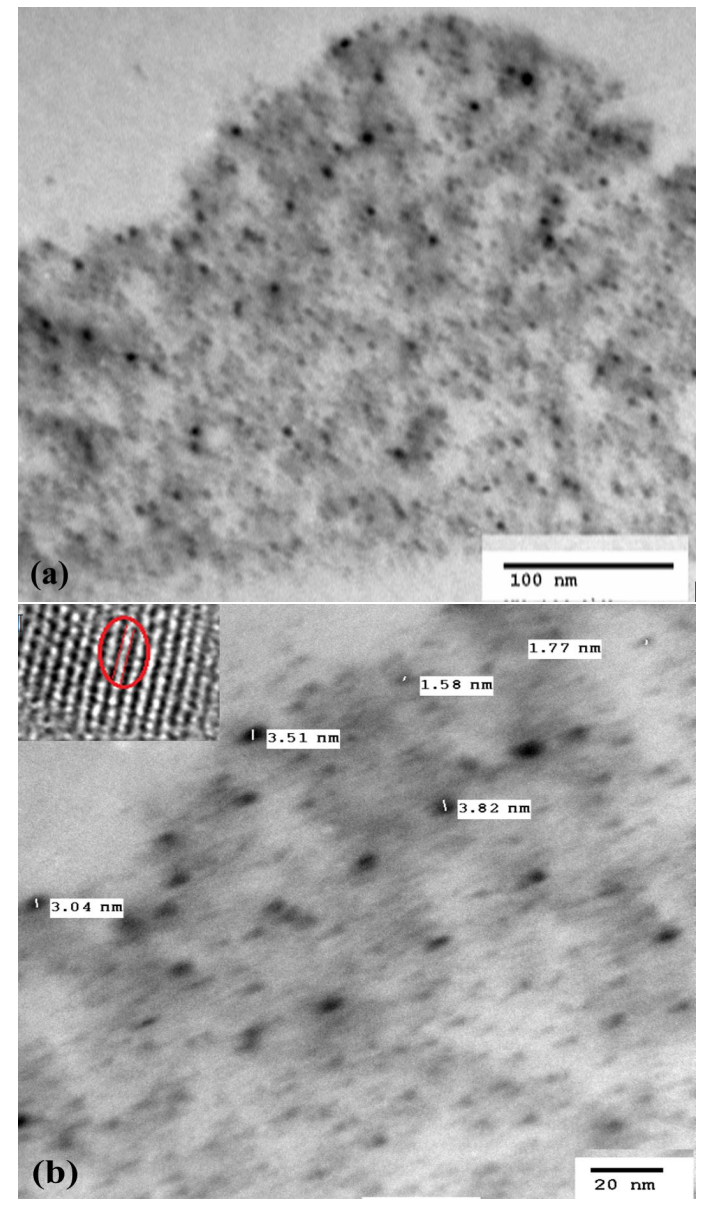

Fig. 3. (a) TEM images of oleic acid modified CdTe nanocrystals at $100 \mathrm{~nm}$ scale (b) $20 \mathrm{~nm}$ scale the inset: HR TEM showing interplanar spacing.

of polycrystalline cubic zinc blende CdTe. The most distinct feature of synthesized CdTe nanoparticles which sets them apart from previous studies is its absorbance peak and specific PL behavior. UV-Vis spectroscopy measurements were conducted with a spectrophotometer in the wavelength range of $800 \mathrm{~nm}$ to $200 \mathrm{~nm}$ using a quartz cuvette and the corresponding results are shown in Fig. 4. The absorbance spectra shown in Fig. 4 exhibit a peak at $570 \mathrm{~nm}$. Different applications of this material which depend on the direct or indirect band gap values could be expressed by the relation between absorption coefficient $(\alpha)$ and incident photon energy ( $\mathrm{h} v$ ) as shown in equation 1 :

$$
a=\frac{A\left(h v-E_{g}\right)^{n}}{h v}
$$

where $\mathrm{A}$ is the constant and $\mathrm{E}_{\mathrm{g}}$ is the band gap of the material. The exponent $\mathrm{n}$ depends on the type of transition; its value is $1 / 2$ for direct band gap and 2 for indirect band gap.

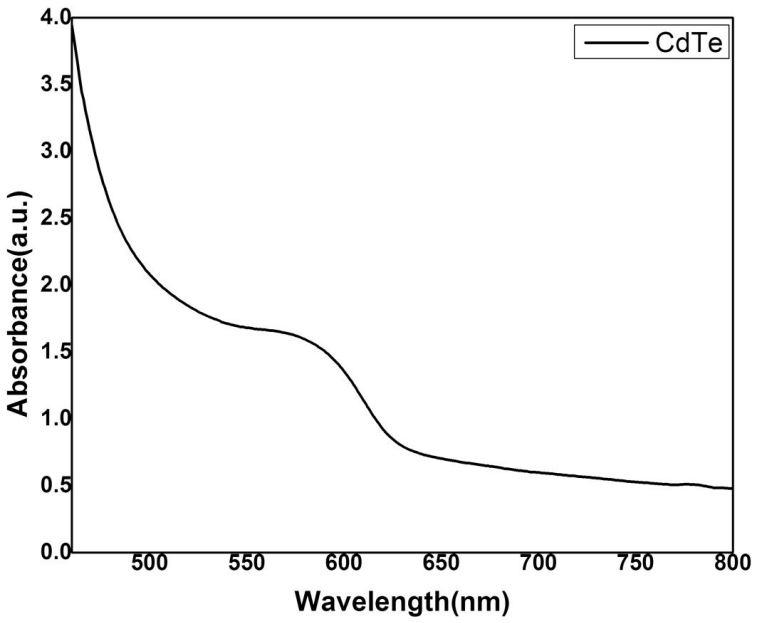

Fig. 4. Absorption spectra of oleic acid capped CdTe nanocrystals in ODE solution.

The exact value of direct or indirect bandgap could be determined from the broad absorption peak by extrapolating the straight line portion of the $(\alpha h v)^{2}$ versus hv graph (Fig. 5). The higher band gap $(2.7 \mathrm{eV})$ as compared to its bulk value found from the Tauc plot (Fig. 5), is due to the smaller quantum dot size leading to the effective quantum confinement. The tunable size enables to tune the absorption and emission properties of QDs which is immensely beneficial for QD analysis. The size of quantum dots, D was calculated using the expression shown equation 2 [20]:

$$
\begin{aligned}
& D[\mathrm{~nm}]=59.60816-0.54736 \lambda+1.8873 \times 10^{-3} \times \lambda^{2} \\
& -2.85743 \times 10^{-6} \times \lambda^{3}+1.62974 \times 10^{-9} \times \lambda^{4}
\end{aligned}
$$

where $\lambda$ is the wavelength. Here, the exciton peak position is at $\lambda=570 \mathrm{~nm}$ and the size of quantum dots was estimated as $3.65 \mathrm{~nm}$ which is in good agreement with the size derived from TEM image. The quantum dot size assuming exciton confinement in particle was further calculated using effective mass approximation (EMA) with the relation 
between optical band gap and particle size corelated with the equation 3 [21]:

$$
E_{g}=E_{\text {bulk }}+\frac{h^{2}}{8 r^{2}}\left(\frac{1}{m_{e}^{*}}+\frac{1}{m_{h}^{*}}\right)-\frac{e^{2}}{4 \pi \varepsilon_{0} \varepsilon_{r} \gamma_{e}}
$$

where $E_{g}$ and $E_{\text {bulk }}$ are the band gaps of nano and bulk CdTe, respectively, $\mathrm{m}_{\mathrm{e}}^{*}$ is the effective electron mass of CdTe $\left(0.11 \mathrm{~m}_{\mathrm{e}}\right), \mathrm{m}_{\mathrm{h}}^{*}$ is the effective hole mass of CdTe $\left(0.4 \mathrm{~m}_{\mathrm{e}}\right), \varepsilon_{0}\left(8.854 \times 10^{-12}\right)$ is the dielectric constant of air, $\varepsilon_{\mathrm{r}}(10.25)$ is the relative dielectric constant and $\gamma_{\mathrm{e}}$ is the Bohr excitation radius. The calculations resulted in the radius of CdTe quantum dot as $1.81 \mathrm{~nm}$ and the size of quantum dot as $3.62 \mathrm{~nm}(2 \mathrm{r})$. The average QD size approximated both from semi-empirical $(3.65 \mathrm{~nm})$ and EMA $(3.62 \mathrm{~nm})$ methods are in good agreement with the size estimated from TEM $(3.5 \mathrm{~nm})$ investigation.

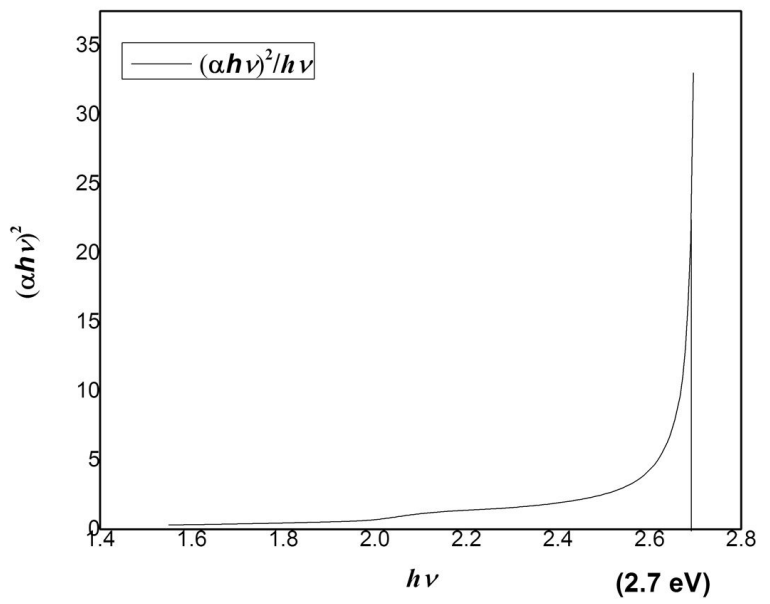

Fig. 5. Band gap calculated from the Tauc plot $\left((\alpha \mathrm{h} \vartheta)^{2}\right.$

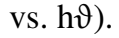

The emission spectrum of oleic acid caped CdTe quantum dots was recorded by exciting at $550 \mathrm{~nm}$ and the curve is shown in Fig. 6. The spectrum shows a broad emission at visible region having emission maxima at $650 \mathrm{~nm}$. Thus, orange light is likely to originate from the radiative recombination of electron-hole pairs produced in these particles. It is also important to note that the separation between absorption and emission peak, i.e. Stokes shift which was thought to arise is a clear indication of the pure band edge emission without

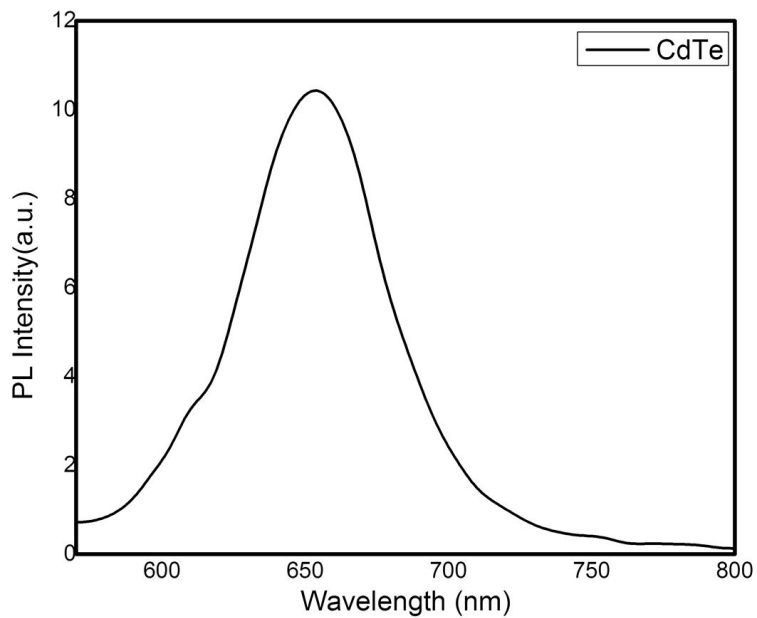

Fig. 6. Fluorescence spectrum of oleic acid modified CdTe QD excited at $550 \mathrm{~nm}$.

any significant trap-state emission [22]. Nevertheless, the smaller overlap area between the absorption and emission spectra could be favorable for optoelectronic applications [23]. The surface passivation of oleic acid stabilized CdTe nanoparticles was further characterized by FT-IR and shown in Fig. 7. The appearance of the peaks at $1520 \mathrm{~cm}^{-1}$, $1700 \mathrm{~cm}^{-1}$ and $1060 \mathrm{~cm}^{-1}$ could be attributed to $\mathrm{C}=\mathrm{C}, \mathrm{C}=\mathrm{O}$ and $\mathrm{C}-\mathrm{O}$, respectively $[24,25]$. The peaks at $2904 \mathrm{~cm}^{-1}$ and $770 \mathrm{~cm}^{-1}$ ascribed to the long alkyl chain of corresponding oleic acid have appeared. The peak at $3707 \mathrm{~cm}^{-1}$ could be connected with the $\mathrm{OH}$ of unreacted oleic acid on $\mathrm{CdTe}$ surface. In order to observe the quenching effect of CdTe quantum dots, the PL experiment was conducted at continuous addition of quantum dot solution of concentration $10^{-6} \mathrm{M}(100 \mu \mathrm{L}$ at a slot $)$ to a solution of $1.5 \times 10^{-6} \mathrm{M}$ PFNA (Fig. 1) and with a mild sonication excited at $350 \mathrm{~nm}$. Fig. 8 shows the quenching of PL intensity of conjugated polymer (PFNA) on addition of oleic acid capped CdTe nanoparticles.

The quenching is quite efficient till the addition of $500 \mu \mathrm{L}$ of the quantum dot solution and then the rate of quenching slows down. The observed quenching may arise after diffusion of quantum dot in polymer surface and may result from the coordination ability of pyridine containing homopolymer with CdTe quantum dot [26]. The drastic 


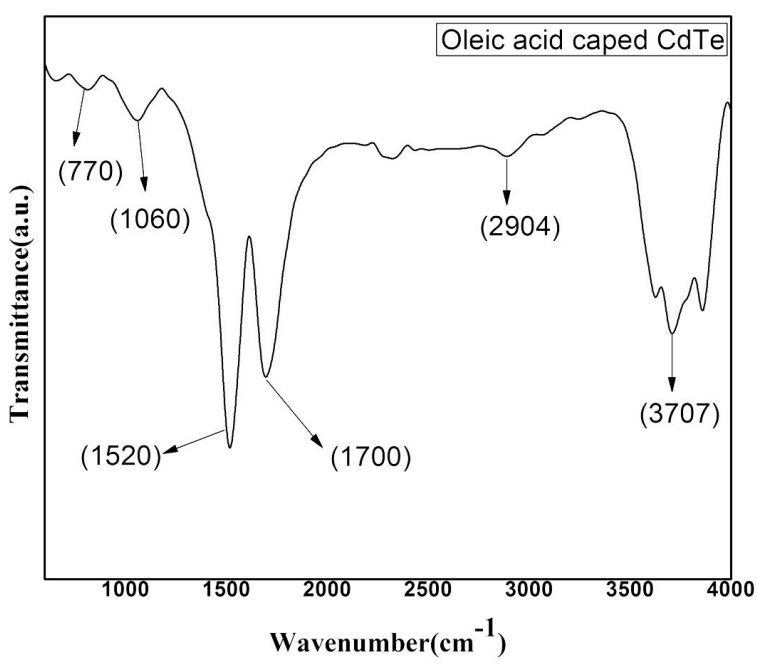

Fig. 7. FT-IR spectra of oleic acid caped CdTe quantum dots at room temperature.

quenching may occur when the CdTe quantum dots get encapsulated within the hydrophobic domain of the polymer; however there is no significant change in the wavelength.

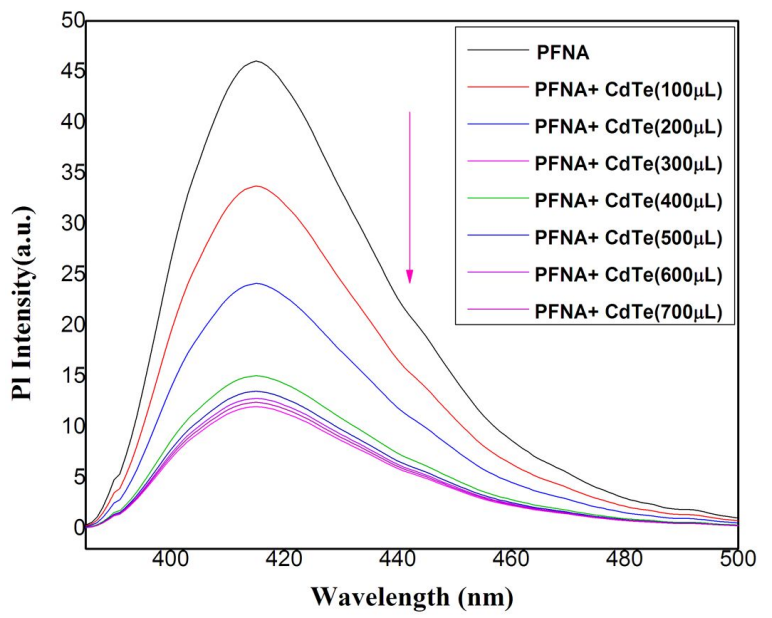

Fig. 8. PL measurement of polymer(PFNA) solutions on addition of CdTe quantum dot.

\section{Conclusion}

We synthesized oleic acid modified CdTe quantum dots through hot-injection with oleic acid as the surface ligand and ODE as the solvent. The formation of surface passivated zinc blend structure of CdTe has been well characterized by FT-IR and XRD spectroscopy. The structure and size of the CdTe nanocrystals were obtained from XRD and HR-TEM measurements and exhibited a cubic zinc blende structure with the particle size of $3 \mathrm{~nm}$ to $4 \mathrm{~nm}$. Furthermore, the average QD size approximated both from semi-empirical and EMA method was in good agreement with the size estimated from TEM investigation. A simple strategy for semiconducting PFNA-CdTe nanocomposite polymer was then explored by sequential addition of CdTe QD into PFNA solution. The gradual addition of CdTe QD into PFNA solution caused the photoluminescence quenching of the homopolymer (PFNA). The coordination of pyridine containing homopolymer with CdTe quantum dot may find better interest to the diverse readership of material research.

\section{Acknowledgements}

We are grateful to the National Institute of Science and Technology for financial support for this work. The authors would also like to thank Dr. Kui-Hsien Chen, IAMS, Academia Sinica, Taiwan, and Dr. K.K. Nanda, Materials Research Center, IISc, Bangalore, for providing some of the structural and optical characterization facilities.

\section{References}

[1] Zhao T., Goodwin E D., Guo J., Wang H., Diroll B.T, Murray C.B., Kagan C.R., ACS Nano, 10 (2016), 9267.

[2] Houtepen A.J., Hens Z., Owen J.S., Infante I., Chem. Mater., 2 (2017), 752.

[3] Yukawa H., BabA Y., Anal. Chem., 5 (2017), 2671.

[4] Yue Z., Lisdat F., Parak W.J., Hickey S.G., Tu L., Sabir N., Dorfs D., Bigall N.C., ACS Appl. Mater. Interfaces, 8 (2013), 2800.

[5] Tiwari A., Dhoble S.J., Cryst. Growth Des., 1 (2017), 381.

[6] Rao C N.R., Matte H.S.S.R., VogGu R., GovinDARAJ A., Dalton Trans., 17 (2012), 5089.

[7] Xu S., Lu H., Li J., Song X., Wang A., Chen L., HAN S., ACS Appl. Mater. Interfaces, 16 (2013), 8146.

[8] Hühn J., Carrion C.C., Soliman M.G., Pfeiffer C., Valdeperez D., Masood A., Chakraborty I., Zhu L., Gallego M., Yue Z., Carril M., Feliu N., Escudero A., Alkilany A.M., Pelaz B., Pino P.D., PARAK W.J., Chem. Mater., 1 (2017), 399.

[9] Hetsch F., Xu X., Wang H., Kershaw S.V., RoGACH A.L., J. Phys. Chem. Lett., 15 (2011), 1879.

[10] Wang K., Zhang R., Sun N., Li X., Wang J., CaO Y., Pei R., ACS Appl. Mater. Interfaces, 39 (2016), 25834 . 
[11] Islam M.A., Huda Q., Hossain M.S., Aliyu M.M., KARIM M.R., Sopian K., Amin N., Cur. Appl. Phys., 13 (2013), 115.

[12] Gutiérrez-Lazos C.D., Ortega-López M., PÉreZ-GuzMán M.A., EsPinOZA-Rivas A.M., SOlís-Pomar F., ORTEGA-AMAYA R., SilvaVidaurri L.G., CAstro-Peña V.C., PÉrezTiJERINA E., Beilstein J. Nanotechnol., 5 (2014), 881.

[13] Jung J., Pang X., Feng C., Lin Z., Langmuir, 25 (2013), 8086.

[14] Han J., Zhou Z., Bu X., Zhu S., Zhang H., Sun H., YANG B., Analyst, 12 (2013), 3402.

[15] BaZargan A.M., Sharif F., Mazinani S., Naderi N., J. Mater. Sci.: Mat. Electron., 8 (2016), 8221.

[16] Sahu D., Chu H.C., YAng P.J., Lin H.C., Macromol. Chem. Phys., 15 (2012), 1550.

[17] Sahu D., Chakraborty S., Mater. Sci.-Poland, 1 (2017), 217.

[18] Shen M., Jia W., You Y., Hu Y., Li F., Tian S., Li J., Jin Y., HAN D., Nanoscale Res. Lett., 1 (2013), 253.
[19] WANG Y., WANG R., LiU S., YANG K., ZhOU L., LI H., Bull. Chem. Soc. Ethiop., 3 (2013), 387.

[20] Yu Jasieniak J., Smith L., Embden J.V., MulVAne P., J. Phys. Chem. C, 113 (2009), 19468.

[21] Nemade K.R., Waghuley S.A., Res. Phys., 3 (2013), 52.

[22] Mohanta D., Narayanan S.S., Pal S.K., RaYCHAUdHURi A.K., J. Exp. Nanosci., 4 (2009), 177.

[23] Kim D.J., Koo K.K., Cryst. Growth Des., 2 (2009), 1153.

[24] Depalo N., Comparelli R., Huskens J., Ludden M.J.W., Perl A., Agostiano A., Striccoli M., CURRI M.L., Langmuir, 23 (2012), 8711.

[25] Baoqing S., Jipeng L., Zhixue W., Chinese J. Chem. Eng., 6 (2006), 814.

[26] Wang M., Felorzabihi N., Guerin G., HaLEY J.C., Scholes G.D., WinNiK M.A., Macromolecules, 17 (2007), 6377.

Received 2018-07-13 Accepted 2019-04-23 